\title{
Nonlinear Phase Separation Testing of an Experimental Wing-Engine Structure
}

\author{
L. Renson ${ }^{1}$, J.P. Noël' ${ }^{2}$, D.A.W. Barton ${ }^{1}$, S.A. Neild ${ }^{1}$, G. Kerschen ${ }^{2}$ \\ ${ }^{1}$ Faculty of Engineering, University of Bristol, Bristol, UK. \\ ${ }^{2}$ Department of Aerospace and Mechanical Engineering, University of Liège, Liège, Belgium.
}

\begin{abstract}
Modal testing of nonlinear engineering structures is currently a research area that attracts substantial attention. Recently, a nonlinear generalisation of phase separation testing has been proposed for identifying nonlinear normal modes (NNMs) based on input and output measurements. The nonlinear phase separation (NPS) method integrates identification and continuation tools to calculate multiple NNMs from broadband data. The present contribution reports the first experimental application of the NPS method. The structure of interest is a model inspired by a wing with two engines connected by means of nonlinear pylons with softening-hardening stiffness characteristics. The frequency-energy dependences of four NNMs in the $0-100 \mathrm{~Hz}$ range are estimated, including modal interactions. NNMs identified using the NPS method are also compared to free-decay testing results.
\end{abstract}

Keywords: nonlinear normal modes, experimental identification, broadband excitation, experimental model, discretetime continuation.

Experimental linear modal analysis (EMA) is routinely practiced in industry and plays an important role in the development and certification of aerospace structures. For instance, the resonance frequencies, vibration modes and damping ratios identified from experimental tests are used to update and validate mathematical models that can be used for flutter predictions. However, the increasing presence of nonlinearity arising, for example, from large displacements, joints or complex material properties, poses a number of issues leading to erroneous identification results ${ }^{[1]}$.

Nonlinear normal modes (NNMs) offer a theoretical framework to extend the concept of modal analysis to nonlinear systems. NNMs can explain important nonlinear phenomena such as mode bifurcations and interactions between modes with wellseparated, non-commensurate natural frequencies. The most popular definition defines NNMs as periodic solutions of the unforced, undamped equations of motion of the system in question. The experimental identification of such periodic motions can be performed by applying an excitation, multi-harmonic and distributed across the structure as appropriate, in phase quadrature with the response of the structure. At quadrature, the applied excitation counterbalances exactly the damping forces present in the system and the dynamics of the unforced, undamped system is recovered ${ }^{[2]}$. Such an approach follows the philosophy of linear phase resonance (force appropriation) techniques to extract each NNM individually.

A nonlinear phase separation (NPS) technique where multiple NNMs are extracted from broadband data collected during a single experiment was proposed and demonstrated on a nonlinear beam using simulated data ${ }^{[3]}$. The present paper improves this NPS methodology and reports its first experimental demonstration.

The structure of interest for this study is shown in Figure 1 (a). It consists of a continuous $1 \mathrm{~m}$-long, $5 \mathrm{~mm}$-thick aluminum plate (the wing) to which two masses of $1.38 \mathrm{~kg}$ (the engines) are suspended. The suspension of each mass is made of two vertical thin beams, one at the front and one at the back of each mass. The beams are bolted to the mass at one end and clamped to the wing at the other end. The surface of the clamps is curved such that the effective length of the suspension beams is a function of the deflection amplitude, hence providing nonlinearity. The whole structure is suspended on soft springs such that rigid-body-like modes are restrained below $5 \mathrm{~Hz}$. The reader is referred to Londoño et al ${ }^{[4]}$ for further details about the experimental setup. 


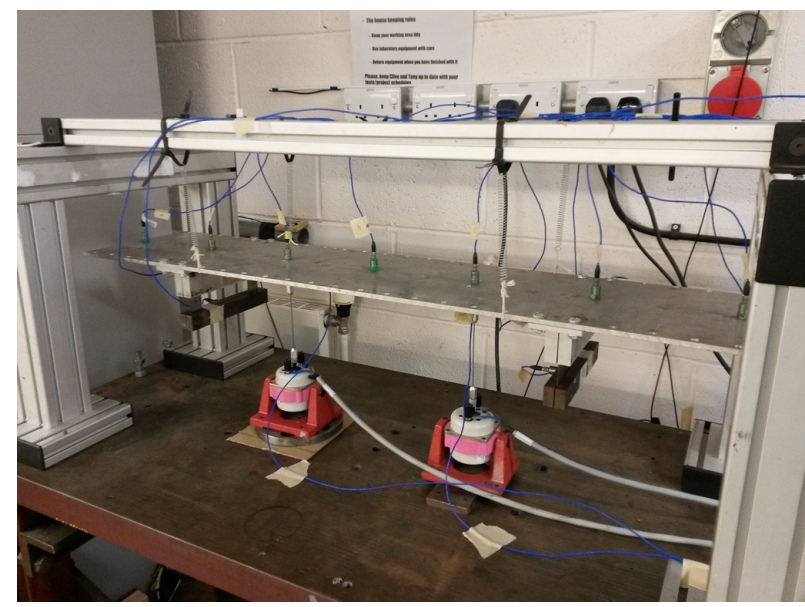

(a)

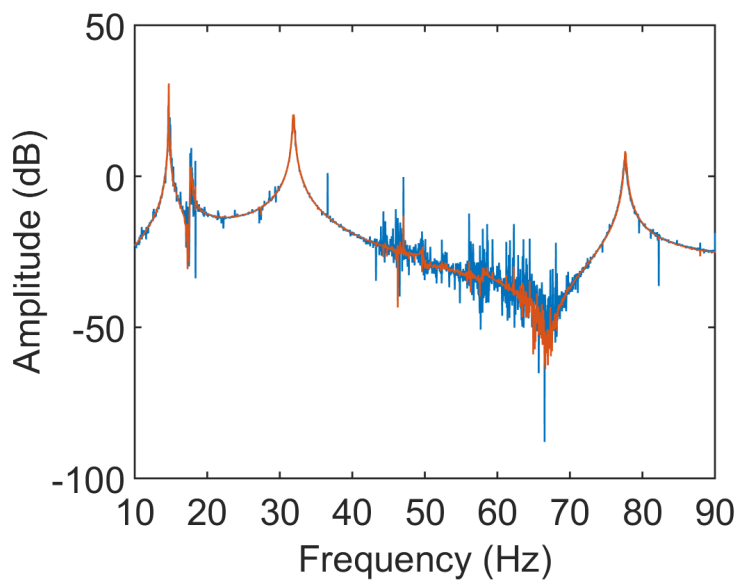

(b)

Figure 1: (a) Picture of the Wing-Engine structure. (b) FRF of the structure at $0.25 \mathrm{~V}(-)$ and $1.25 \mathrm{~V}(-)$.

Figure $1(\mathrm{~b})$ presents the frequency response functions (FRFs) of the system collected at $0.25 \mathrm{~V}(-)$ and $1.25 \mathrm{~V}(-)$. The structure presents 4 modes between $10-90 \mathrm{~Hz}$. The FRF measured at high level shows clear signs of nonlinear distortions. The peaks corresponding to the first two modes shift towards higher frequencies and the whole FRF is altered by nonlinear stochastic distortions.

The first and second modes have mode shapes in which the two masses move out-of- and in-phase respectively and are the most affected by nonlinearity. As such, sine-sweep data were also collected at different excitation levels around these two modes. The sweeps were performed at a linear rate of $5 \mathrm{~Hz}$ per minute between $10 \mathrm{~Hz}$ and $25 \mathrm{~Hz}$. The restoring force surface (RFS) method was applied to the time series around the first mode in order to characterise the form of the nonlinearity. The nonlinear stiffness curve obtained for each connection is smooth as shown in Figure 2(a). The time-frequency content of the high-level sine sweep data were also analyzed using the wavelet transform, revealing the presence of a 5:1 modal interaction between the first and fourth modes of the structure (see Figure 2(b)).

The NPS method comprises two main steps. The first one consists of processing the acquired broadband input and output data to derive an experimental model of the structure. To this end, a frequency-domain subspace identification (FNSI) method is used $^{[5]}$, although any method applicable to broadband data can be employed. The second step of the identification methodology is to extract the NNMs from the identified model using numerical continuation techniques ${ }^{[6]}$.

The broadband input is a pseudo-random signal (multisine with random phases) defined between $10 \mathrm{~Hz}$ and $90 \mathrm{~Hz}$ in order to encompass the four modes of interest and the fundamental harmonics of the first two modes. The random signal is repeated 15 times and the last 10 periods are considered for averaging. The structure response is recorded through 11 accelerometers. The model provided by FNSI is a discrete-time state-space model of the form

$$
\left\{\begin{array}{l}
\mathbf{x}_{k+1}=\mathbf{A} \mathbf{x}_{k}+\mathbf{B e}\left(\mathbf{y}_{k}, \mathbf{u}_{k}\right) \\
\mathbf{y}_{k}=\mathbf{C} \mathbf{x}_{k}+\mathbf{D e}\left(\mathbf{y}_{k}, \mathbf{u}_{k}\right)
\end{array}\right.
$$

where the matrices $(\mathbf{A}, \mathbf{B}, \mathbf{C}, \mathbf{D})$ are the state, extended input, output and direct feedthrough matrices, respectively. The vectors $\mathbf{x}, \mathbf{y}$, and $\mathbf{u}$ are the states, outputs, and external inputs of the system, respectively. The vector e, termed the extended input vector, concatenates the external forces and the nonlinear basis functions used to model the nonlinearities. Considering the smoothness of the stiffness curves observed in Figure 2(a), smooth basis functions such as polynomials (cubic) and splines are considered to model the nonlinearity.

Noël et al. ${ }^{[3]}$ considered the transformation of Eqs. (1) into continuous time and its projection onto the linear modal basis in order to easily remove damping terms and carry out the computation of the NNMs in a conservative setting. In the present paper, the original discrete-time model (1) provided by FNSI is kept. An single-point external harmonic excitation is applied to the damped 


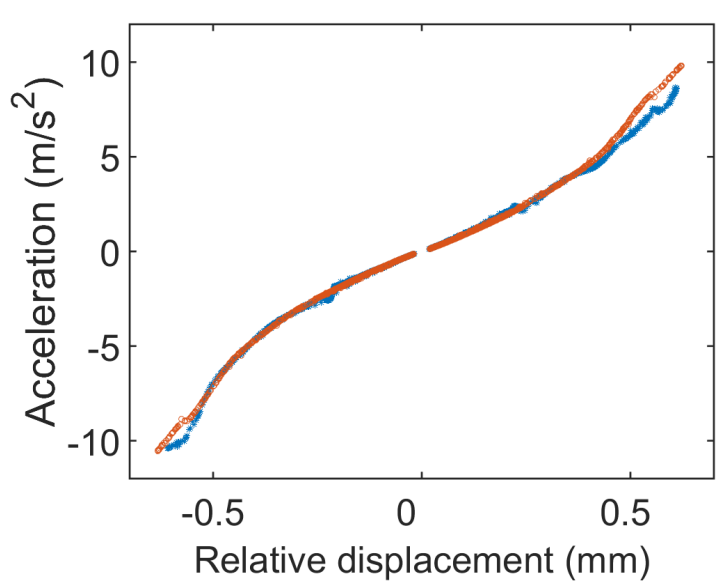

(a)

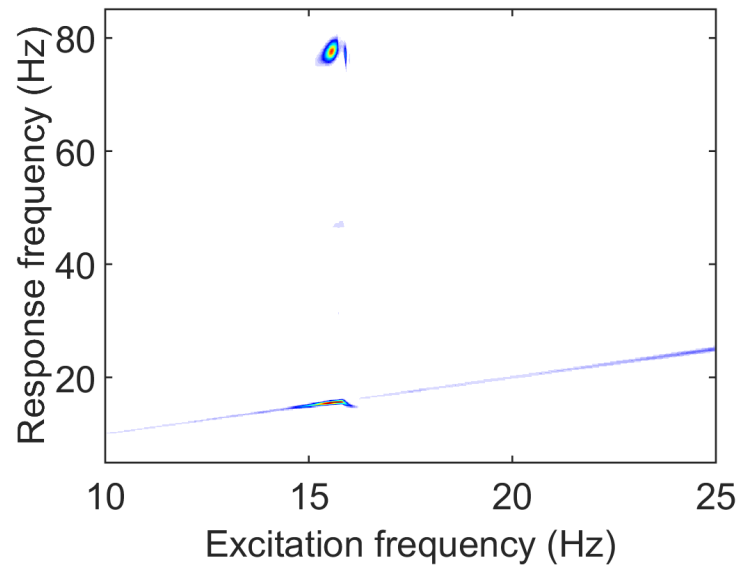

(b)

Figure 2: (a) Qualitative restoring force measured at the right $(-)$ and left $(-)$ nonlinear connections. (b) Wavelet transform analysis of the sine-sweep data collected at a high level of excitation.

system. The excitation is of the form $u_{k}=F \cos (2 \pi k h / T)$ where $F$ and $T$ are the amplitude and period of forcing, and $h$ is the sampling time used to collect experimental data. Numerical continuation is used to track the forced response of the system. To extract the NNMs, an additional quadrature condition between the phase of the response and the excitation is imposed and a two-parameter continuation in $(F, T)$ is carried out.

The NNMs extracted using the NPS method are analyzed and compared to resonant decay results ${ }^{[4]}$. The reproduction by the first NNM of the modal interaction observed in the sine sweep data is discussed. Additional comparisons between the nonlinear forced responses calculated from Eqs. (1) and the sine sweep data are also carried out.

\section{References}

[1] B. Peeters, H. Van der Auweraer, Application of multisine excitation to aircraft Ground Vibration Testing, In Proceedings of the 16th IFAC Symposium on System Identification, Brussels, Belgium, 2012.

[2] M. Peeters, G. Kerschen, and J. C. Golinval. Modal testing of nonlinear vibrating structures based on nonlinear normal modes: Experimental demonstration. Mechanical Systems and Signal Processing, 25(4):1227-1247, 2011.

[3] J.P. Noël, L. Renson, C. Grappasonni, G. Kerschen, Identification of nonlinear normal modes of engineering structures under broadband forcing, Mechanical Systems and Signal Processing, 74:95-110, 2016.

[4] J. M. Londoño, S. A. Neild, J. E. Cooper, Identification of backbone curves of nonlinear systems from resonance decay responses, Journal of Sound and Vibration, 348:224-238, 2015.

[5] J.P. Noël, G. Kerschen, Frequency-domain subspace identification for nonlinear mechanical systems, Mechanical Systems and Signal Processing, 40:701-717, 2013.

[6] L. Renson, G. Kerschen, B. Cochelin, Numerical computation of nonlinear normal modes in mechanical engineering, Journal of Sound and Vibration, 364:177-206, 2016. 